\title{
Endometriosis-Related Pain Reduction During Bleeding and Nonbleeding Days in Women Treated with Elagolix
}

This article was published in the following Dove Press journal: Journal of Pain Research

\author{
Sanjay K Agarwal $\mathbb{D}^{\prime}$ \\ Sukhbir S Singh $\mathbb{D}^{2}$ \\ David F Archer $\mathbb{D D}^{3}$ \\ Yabing Mai $\mathbb{D}^{4}$ \\ Kristof Chwalisz ${ }^{5}$ \\ Keith Gordon ${ }^{6}$ \\ Eric Surrey ${ }^{7}$
}

'Department of Obstetrics and Gynecology and Reproductive Sciences, Center for Endometriosis Research and Treatment, UC San Diego, La Jolla, CA, USA; ${ }^{2}$ Clinical Epidemiology Program, Ottawa Hospital Research Institute, Ottawa, Ontario, Canada; ${ }^{3}$ Department of Obstetrics and Gynecology, Eastern Virginia Medical School, Norfolk, VA, USA; ${ }^{4}$ Statistics, AbbVie Inc, North Chicago, IL, USA; ${ }^{5}$ Clinical Development, AbbVie Inc, North Chicago, IL, USA; ${ }^{6}$ Medical Affairs, AbbVie Inc, North Chicago, IL, USA; ${ }^{7}$ Colorado Center for Reproductive Medicine, Lone Tree, CO, USA
Correspondence: Sanjay K Agarwal Department of Obstetrics and Gynecology and Reproductive Sciences, Center for Endometriosis Research and Treatment, UC San Diego, 9500 Gilman

Drive, \#0633, La Jolla, CA, USA

Tel + I (858) 534-8930

Email skagarwal@ucsd.edu
Objective: In this post hoc analysis, we evaluated the impact of elagolix on dysmenorrhea and nonmenstrual pelvic pain across menstrual period (bleeding days) and nonmenstrual (nonbleeding) days.

Methods: Data from two randomized, 6-month, placebo-controlled trials (Elaris Endometriosis (EM)-I and EM-II) of elagolix (150 mg once daily (QD) and $200 \mathrm{mg}$ twice daily (BID)) in premenopausal women with moderate to severe endometriosis-associated pain $(\mathrm{N}=1686)$ were pooled. Women recorded the presence of menstrual period and severity of dysmenorrhea or nonmenstrual pelvic pain in a daily electronic diary.

Results: At baseline, women in the placebo group and both elagolix treatment groups reported moderate or severe dysmenorrhea, on average, $81 \%$ of their menstrual period days and moderate/ severe nonmenstrual pelvic pain, on average, $56 \%$ of their nonmenstrual (nonbleeding) days. Compared with placebo at month 6 , elagolix-treated women had a significantly lower mean (standard deviation (SD)) percentage of menstrual period days with moderate or severe dysmenorrhea (elagolix $150 \mathrm{mg}$ QD = 52.4 (38.9), $\mathrm{p}=0.002$; elagolix $200 \mathrm{mg} \mathrm{BID}=38.5$ (43.6), $\mathrm{p}<$ 0.001 , placebo $=61.3(33.7))$ and a significantly lower mean (SD) percentage of nonmenstrual (nonbleeding) days with moderate or severe nonmenstrual pelvic pain (elagolix $150 \mathrm{mg}$ QD = 31.1 (35.8), $\mathrm{p}<0.001$; elagolix $200 \mathrm{mg}$ BID = 19.7 (29.9), $\mathrm{p}<0.001$; placebo = 35.6 (33.9)).

Conclusion: Following 6 months of elagolix treatment, women who still menstruated had a lower proportion of menstrual period days with moderate or severe dysmenorrhea compared with placebo, demonstrating pain reduction despite continued menses. Additionally, pain did not shift from dysmenorrhea to nonmenstrual pelvic pain, as the percentage of days with moderate or severe nonmenstrual pelvic pain was also reduced for elagolix-treated women compared with placebo.

Trial Registration: The Elaris EM-I study is registered with the US National Library of Medicine, www.ClinicalTrials.gov, NCT01620528. The Elaris EM-II study is registered with the US National Library of Medicine, www.ClinicalTrials.gov, NCT01931670. Both studies are registered with the EU Clinical Trial Register, www.clinicaltrialsregister.ed, 2011004295-11.

Keywords: bleeding, dysmenorrhea, elagolix, endometriosis, nonmenstrual pelvic pain

\section{Introduction}

Endometriosis is an estrogen-dependent disease that affects between $6 \%$ and $10 \%$ of women of reproductive age. ${ }^{1}$ Endometriosis-associated pain symptoms include, but are not limited to, pain during menstruation (dysmenorrhea), pelvic pain not associated with menstruation (nonmenstrual pelvic pain), and pain during 
intercourse (dyspareunia). ${ }^{2-4}$ Most women with endometriosis who experience intense pain around menses have pain that begins approximately 3 to 4 days prior to the start of menses and lasts until the end of menses. ${ }^{5}$ Women who experience both dysmenorrhea and nonmenstrual pelvic pain can be left with almost no reprieve from pain. Chronic pelvic pain, including both dysmenorrhea and nonmenstrual pelvic pain, is one of the most common presenting symptoms in women with endometriosis, occurring in up to $98 \%$ of patients. ${ }^{6}$ These pain symptoms can be debilitating and negatively impact a woman's quality of life. ${ }^{7,8}$

Pain is complex and with women experiencing both pain during bleeding and pain that is not timed with bleeding, it is necessary that medical management of endometriosis addresses both forms of pain. Bleeding suppression or amenorrhea has traditionally been the goal of medical management to treat endometriosis-associated pain, reflecting the efficacy of therapy. Current therapies used in medical management have been shown to suppress bleeding and reduce dysmenorrhea. However, these therapies are either ineffective in reducing nonmenstrual pelvic pain or are associated with side effects not conducive to long-term use. ${ }^{9,10}$ Levonorgestrel-releasing intrauterine devices have been shown to eliminate dysmenorrhea by eliminating menses. However, this therapy is designed to work in the endometrium and adjacent tissues and, therefore, may not address nonmenstrual pelvic pain. ${ }^{9}$ Gonadotropin-releasing hormone $(\mathrm{GnRH})$ agonists suppress menses and reduce dysmenorrhea. While effective in reducing endometriosis-associated nonmenstrual pelvic pain, GnRH agonists are associated with hypoestrogenic effects and are known to cause up to a 3-month delay in the return of regular menses (package insert depot formulation); thus, there is a delay in potential conception. $^{10}$

Recently, elagolix, an oral GnRH antagonist, was approved in the United States and Canada to manage moderate-to-severe pain associated with endometriosis. Results from two placebo-controlled, Phase III studies (Elaris Endometriosis I (EM-I) and Elaris Endometriosis II (EM-II)) demonstrated that 6 months of elagolix treatment with either $150 \mathrm{mg}$ once daily (QD) or $200 \mathrm{mg}$ twice daily (BID) doses resulted in clinically significant dosedependent reductions in dysmenorrhea and nonmenstrual pelvic pain in women with moderate-to-severe endometriosis-associated pain. ${ }^{11}$

Varying degrees of suppression of the hypothalamic pituitary ovarian axis are also seen with elagolix therapy.
At $200 \mathrm{mg}$ BID, there is nearly full estrogen suppression, yet some women continue to bleed. The lower dose of elagolix (150 mg QD) is less likely to induce amenorrhea. $^{12}$ In the Elaris EM-I and EM-II studies, a higher proportion of women treated with elagolix were amenorrhoeic after 6 months of treatment compared with women receiving placebo. ${ }^{11}$ Therefore, it remains undetermined as to whether the reduction in dysmenorrhea in women treated with elagolix is due to increased amenorrhea, with dysmenorrhea in these women shifting to nonmenstrual pelvic pain, or a genuine reduction in pain during bleeding in women treated with elagolix who continue to have bleeding days.

Regarding linzagolix, another oral GnRH antagonist currently in clinical development, similar uncertainty exists as to whether its effectiveness is attributed to amenorrhea rates. ${ }^{13}$ In a recent study, reductions in dysmenorrhea and nonmenstrual pelvic pain were observed with linzagolix treatment, along with an increase in rates of amenorrhea. $^{13}$

This post hoc analysis aims to address the impact of elagolix on nonmenstrual pelvic pain and to ascertain whether suppression of bleeding is required for pain relief in women enrolled in the double-blind, placebo-controlled, phase 3 Elaris EM-I and Elaris EM-II trials.

\section{Materials and Methods}

Data for this post hoc analysis were obtained from two replicate, double-blind, randomized, placebo-controlled, phase 3, institutional review board (IRB)-approved clinical trials (Elaris EM-I (NCT01620528) and Elaris EM-II (NCT01931670 and 2011-004295-11)) that evaluated the safety and efficacy of elagolix (150 mg QD or $200 \mathrm{mg}$ BID) in women with moderate-to-severe endometriosisassociated pain. Study details and primary results have been previously published. ${ }^{11}$ Participants were premenopausal women, aged between 18 and 49 years, who had received a surgical diagnosis of endometriosis in the previous 10 years and who had moderate or severe endometriosis-associated pain at the time of study entry. Women were excluded if they had a $z$ score of less than -1.5 for bone mineral density at the lumbar spine or femoral neck, or if they had total hip replacement at screening or clinically significant gynecologic conditions or chronic pain conditions unrelated to endometriosis. The Elaris EM-I and Elaris EM-II trials were conducted in accord with International Conference on Harmonization guidelines and applicable regulations and 
ethical principles of the Declaration of Helsinki. Shulman Associates IRB (Cincinnati, $\mathrm{OH}$ ) conducted the majority of the IRB approvals (EM-I/M12-665, IRB approval number 201202559, approval date April 11, 2012; EMII/M12-671, IRB approval number 201208471, approval date November 16, 2012). Informed consent was obtained from all women included in the study prior to enrollment.

Each trial consisted of a washout of hormonal therapies (if applicable); a screening period of up to 100 days, including a minimum of two menstrual cycles; and a 6-month treatment period. During screening, women were switched from the use of their usual analgesic agents to receive permitted protocol-defined rescue therapy with nonsteroidal anti-inflammatory drugs (500-mg naproxen), an opioid of choice by country (eg, 5-mg hydrocodone plus 325-mg acetaminophen), or both. Eligible women were randomly assigned in a $2: 2: 3$ ratio to receive $150 \mathrm{mg}$ of elagolix QD, $200 \mathrm{mg}$ of elagolix BID, or placebo. Women were instructed to use two forms of nonhormonal contraception during the study, which included the following combinations: condom, diaphragm, or cervical cap, all with spermicide; or vaginal sponge impregnated with spermicide and used with a condom. Women were not required to use dual contraception methods if sexual partner(s) were vasectomized at least 6 months prior to screening; the woman had undergone a bilateral tubal occlusion at least 3 months prior to screening; the woman was not sexually active with men; or hormonal contraception was started after completion of month 3. Trial visits were conducted on day 1 (the day of the first study drug dose) and monthly through 6 months.

Women used an electronic diary (e-diary) to report daily pain assessments, rescue analgesic use, and level of bleeding. Dysmenorrhea and nonmenstrual pelvic pain were measured by the e-diary on a 4-point pain-effect scale ranging from 0 (no pain) to 3 (severe pain). Pain experienced by women was assigned to the dysmenorrhea category in the e-diary when subjects reported bleeding and to nonmenstrual pelvic pain when subjects reported no bleeding. Bleeding days were characterized by the days that women responded "yes" to the daily e-diary question, "Did you have your period in the last 24 hours?"

The efficacy endpoints of primary interest to this post hoc analysis were the proportion of responders for nonmenstrual pelvic pain taking into account the use of rescue analgesic medication for endometriosis-associated pain at 3 and 6 months of treatment, as previously described in
Taylor et al, ${ }^{11}$ and the mean number and the mean percentage of patient-reported bleeding and nonbleeding days women experienced mild, moderate, or severe dysmenorrhea or nonmenstrual pelvic pain. The criteria for defining a woman as a responder required both a clinically meaningful reduction in pain based on a response threshold derived using receiver operating characteristics analysis and reduced or stable rescue analgesic use. The receiver operating characteristics analysis defined the threshold for a clinically meaningful change from baseline for both dysmenorrhea and nonmenstrual pelvic pain separately in each trial, based on the use of patient reports of "much improved" and "very much improved" on the Patient Global Impression of Change scale as an anchor; the receiver operating characteristics analysis was described fully by Taylor et al. ${ }^{11}$ Pain scores were averaged over the 35-day window before and including the study visit day.

Baseline analyses included all women who completed the screening period, were randomized, and received at least one dose of study drug. Baseline was defined as the average of the last 35 days during the screening period. Data were pooled across studies and treatment groups. Change-from-baseline results are reported using descriptive statistics. Wilcoxon rank sum tests were used to evaluate differences between elagolix dose groups and placebo. A subgroup contrast within a logistic regression analysis was used to make dose group comparisons for the percent of nonmenstrual pelvic pain responders by bleeding status. The studies were not designed to statistically compare elagolix doses.

\section{Results}

A total of 1686 women were randomized and received at least one dose of elagolix or placebo in the Elaris EM-I (n $=871)$ and Elaris EM-II $(n=815)$ phase 3 trials. At baseline, the mean number of bleeding days was similar across dose groups and ranged from 8.2 to 8.4 days. Women from the placebo groups and both elagolix treatment groups reported having moderate or severe dysmenorrhea on average $81 \%$ of bleeding days. The mean number of nonbleeding days at baseline ranged from 22.5 to 23.1 days across dose groups and women reported having nonmenstrual pelvic pain on average $56 \%$ of these days (Table 1).

While the number of bleeding days at baseline was similar across all dose groups, at months 3 and 6, women treated with elagolix had fewer bleeding days 
Table I Baseline Characteristics for Women Enrolled in Elaris EM-I and EM-II

\begin{tabular}{|c|c|c|c|}
\hline Characteristics & Placebo $\mathbf{N}=734$ & Elagolix I50 mg QD N = 475 & Elagolix $200 \mathrm{mg}$ BID $\mathrm{N}=476$ \\
\hline Age, years & $32 \pm 6.6$ & $32 \pm 6.4$ & $32 \pm 6.6$ \\
\hline BMI, $\mathrm{kg} / \mathrm{m}^{2}$ & $28 \pm 6.3$ & $28 \pm 6.6$ & $27 \pm 6.6$ \\
\hline Dysmenorrhea score (scale, 0-3) & $2.2 \pm 0.5$ & $2.2 \pm 0.5$ & $2.1 \pm 0.5$ \\
\hline No. of bleeding days & $8.4 \pm 3.0$ & $8.1 \pm 2.9$ & $8.2 \pm 3.0$ \\
\hline \multicolumn{4}{|l|}{ Percent of bleeding days with ${ }^{\mathrm{a}}$ : } \\
\hline "None" dysmenorrhea & 1.7 & 2.2 & 2.4 \\
\hline "Mild" dysmenorrhea & 18.0 & 16.3 & 17.9 \\
\hline "Moderate/severe" dysmenorrhea & 80.4 & 81.5 & 79.7 \\
\hline Nonmenstrual Pelvic Pain score $(\text { scale, } 0-3)^{\mathrm{a}}$ & $1.6 \pm 0.5$ & $1.7 \pm 0.5$ & $1.6 \pm 0.5$ \\
\hline No. of nonbleeding days & $22.7 \pm 4.5$ & $23.1 \pm 4.1$ & $22.5 \pm 4.6$ \\
\hline \multicolumn{4}{|l|}{ Percent of nonbleeding days with ${ }^{\mathrm{a}}$ : } \\
\hline "None" nonmenstrual pelvic pain & 9.5 & 9.3 & 10.3 \\
\hline "Mild" nonmenstrual pelvic pain & 34.5 & 31.0 & 35.2 \\
\hline "Moderate/severe" nonmenstrual pelvic pain & 56.0 & 59.6 & 54.6 \\
\hline
\end{tabular}

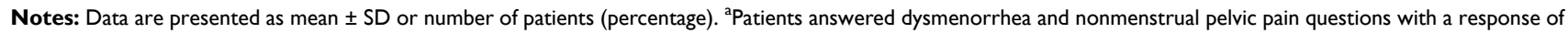
none (0), mild (1), moderate (2), or severe (3).

Abbreviations: BID, twice daily; BMI, body mass index; QD, once daily.

compared with women who received placebo (Figure 1), and a greater proportion of women receiving placebo reported $\geq 1$ day of bleeding compared with women treated with either dose of elagolix.

Across both dose groups, women treated with elagolix experienced fewer days with moderate or severe dysmenorrhea at months 3 and 6 compared with women receiving placebo (Figure 2A). Even though the number of bleeding days decreased with elagolix treatment and conversely the number of nonbleeding days increased, the mean number of nonbleeding days in women with moderate or severe nonmenstrual pelvic pain also declined at month 6 with elagolix treatment (Figure 2C). At month 6, women treated with elagolix reported significantly fewer days with moderate or severe nonmenstrual pelvic pain (month 6 mean (standard deviation $[\mathrm{SD}]$ ) number of days with moderate or severe



Figure I Mean number of bleeding and nonbleeding days at baseline, month 3, and month 6. Missing due to early discontinuation: month 3 (placebo, $0 / 726$; I50 mg elagolix QD, 0/469; $200 \mathrm{mg}$ BID elagolix, I/469) and month 6 (placebo, 8/727; $150 \mathrm{mg}$ elagolix QD, 2/468; $200 \mathrm{mg}$ BID elagolix, 7/468). There were no statistically significant differences between either dose group and placebo for baseline age, body mass index, or mean dysmenorrhea or NMPP scores, except for the dysmenorrhea score in the elagolix $200 \mathrm{mg}$ BID dose group (Elaris EM-II data, $p=0.03$ ).

Abbreviations: BID, twice daily; NMPP, nonmenstrual pelvic pain; QD, once daily. 
A



C

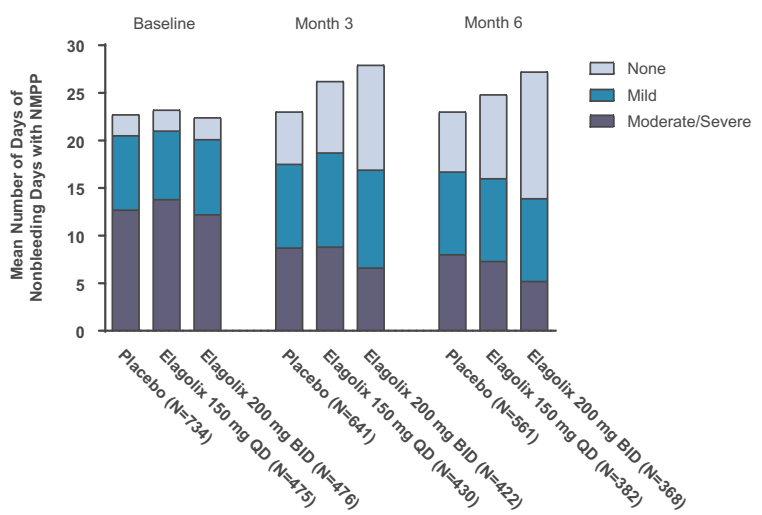

B

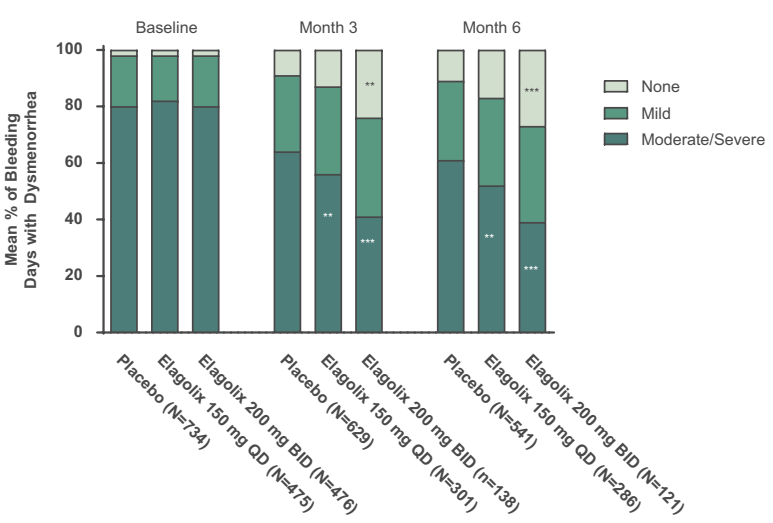

D

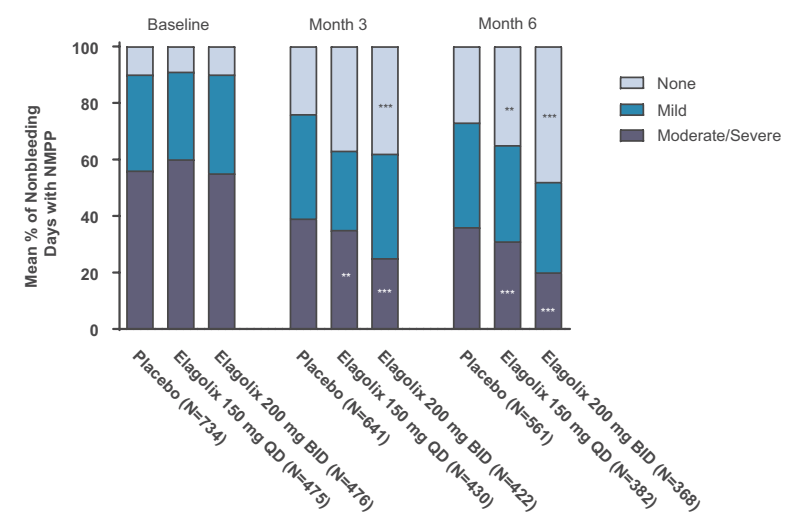

Figure 2 Mean number and mean percent of bleeding days with dysmenorrhea (A and $\mathbf{B})$. Mean number and mean percent of nonbleeding days with nonmenstrual pelvic pain ( $\mathbf{C}$ and D). $\mathrm{P}<0.00 \mathrm{I}\left({ }^{* *}\right)$ and $\mathrm{p}<0.0 \mathrm{I}\left({ }^{* *}\right)$ vs placebo for percent of women with "moderate or severe" or "none" regarding dysmenorrhea or nonmenstrual pelvic pain (NMPP) from a Wilcoxon rank sum test. Number of days is equal to the number of days the subject reported dysmenorrhea or NMPP as none, mild, moderate, or severe. Missing dysmenorrhea with nonmissing NMPP or missing NMPP with nonmissing dysmenorrhea are imputed as zero days. Percentage of days is equal to the number of days the subject reported dysmenorrhea or NMPP divided by the number of days she answered the e-diary, multiplied by 100 . Baseline is defined as the average of the last 35 days prior to and including the first dosing date.

Abbreviations: BID, twice daily; QD, once daily.

nonmenstrual pelvic pain: elagolix $150 \mathrm{mg}$ QD $=7.3(8.9)$, $\mathrm{p}=0.003$; elagolix $200 \mathrm{mg} \mathrm{BID}=5.2$ (8.5), $\mathrm{p}<0.001$; placebo $=8.0(8.3)$ ), and significantly more days without nonmenstrual pelvic pain compared with women taking placebo (month 6 mean (SD) number of days without nonmenstrual pelvic pain: elagolix $150 \mathrm{mg} \mathrm{QD}=8.8$ (10.4), $\mathrm{p}=0.001$; elagolix $200 \mathrm{mg} \mathrm{BID}=13.3$ (12.3), $\mathrm{p}<0.001$; placebo $=6.3(8.4))($ Figure 2C).

Results of further analysis showed that women treated with elagolix who continued to report bleeding, compared with women treated with placebo at months 3 and 6 , had a significantly lower percentage of bleeding days with moderate or severe dysmenorrhea (month 6 mean (SD) percent of bleeding days with moderate or severe dysmenorrhea: elagolix $150 \mathrm{mg}$ QD $=52.4$ (38.9), p = 0.002; elagolix $200 \mathrm{mg} \mathrm{BID}=38.5$ (43.6), $\mathrm{p}<0.001 ;$ placebo $=61.3$ (33.7)) and a significantly lower percentage of nonbleeding days with moderate or severe nonmenstrual pelvic pain (month 6 mean (SD) percent of nonbleeding days with moderate or severe nonmenstrual pelvic pain: elagolix $150 \mathrm{mg}$ QD $=31.1$ (35.8), p < 0.001; elagolix $200 \mathrm{mg}$ $\mathrm{BID}=19.7$ (29.9), $\mathrm{p}<0.001 ;$ placebo $=35.6(33.9))$ (Figure 2B and D). Women treated with elagolix $200 \mathrm{mg}$ BID had a greater percentage of bleeding days with no dysmenorrhea compared with women taking placebo at months 3 and 6 (month 6 mean (SD) percent of bleeding days with no dysmenorrhea: elagolix $150 \mathrm{mg} \mathrm{QD}=16.7$ (30.3), $\mathrm{p}=0.098$; elagolix $200 \mathrm{mg}$ BID = 27.3 (39.9), $\mathrm{p}<$ 0.001 ; placebo $=11.3(22.9)$ ). The percentage of nonbleeding days without nonmenstrual pelvic pain reported was also significantly greater among women treated with elagolix in both dose groups compared with women taking placebo at months 3 and 6 (month 6 mean (SD) percent of nonbleeding days without nonmenstrual pelvic pain: 
elagolix $150 \mathrm{mg}$ QD = 34.8 (37.6), $\mathrm{p}=0.002$; elagolix $200 \mathrm{mg} \mathrm{BID}=47.9$ (40.7), $\mathrm{p}<0.001 ;$ placebo $=27.2$ (33.8)). At both month 3 and month 6 , a significantly greater proportion of women in both elagolix dose groups were nonmenstrual pelvic pain responders compared with women taking placebo, whether they bled or not (Figure 3). At months 3 and 6, a greater proportion of women in both dose groups were dysmenorrhea responders and simultaneously nonmenstrual pelvic pain nonresponders compared with placebo (Table 2).

\section{Discussion}

It was previously reported that 6 months of treatment with either elagolix $150 \mathrm{mg}$ QD or $200 \mathrm{mg}$ BID significantly improved dysmenorrhea and nonmenstrual pelvic pain compared with placebo in women with moderate or severe endometriosis-associated pain. ${ }^{11}$ In the present study, women who continued bleeding while taking elagolix treatment at either $150 \mathrm{mg}$ QD or $200 \mathrm{mg}$ BID had fewer bleeding days with moderate or severe dysmenorrhea. The proportion of nonbleeding days with moderate or severe nonmenstrual pelvic pain was also reduced among women treated with elagolix compared with women receiving placebo following 6 months of treatment.

Overall, pain did not shift from dysmenorrhea to nonmenstrual pelvic pain. This supports the conclusion that with elagolix treatment, women are achieving overall pain relief rather than pain relief due to only the induction of amenorrhea.

Findings from this current study demonstrate for the first time that reduction in pain in women with endometriosis during elagolix treatment occurs during both bleeding and nonbleeding days, suggesting that induction of amenorrhea may not be essential to treat endometriosisrelated pain. A previous clinical study by $\mathrm{Ng}$ et $\mathrm{al}^{14}$ demonstrated that nearly full suppression of estradiol was achieved within hours at elagolix doses of $200 \mathrm{mg}$ BID in 45 patients; however, our study found that some women continued to bleed after 6 months of treatment with elagolix. This apparent difference is likely attributable to incomplete medication compliance in the Phase III Elaris EM-I and EM-II studies, resulting in the continuation of menses. The ability to reduce pain without completely suppressing bleeding with low or high doses of elagolix allows women to not require hypoestrogenism and other adverse effects. These side effects are present with the alternative treatment options that demonstrate a near complete suppression of estrogen. The current findings suggest that the conventional wisdom of associating amenorrhea with symptom relief may not be completely valid. As we turn attention away from suppressing bleeding to reducing pain, we open the way for changes in the medical management of endometriosis-associated pain in women.

Endometriosis-associated pain varies from patient to patient. Pain may be experienced as nonmenstrual pelvic pain or dysmenorrhea, or pain may be related to a specific function, such as defecation (dyschezia), urination (dysuria), or sexual activity (dyspareunia). ${ }^{15}$ The severity of this pain may also fluctuate, and is rarely constant. ${ }^{15}$ It is probable that endometriosis gives rise to multiple different types of pain including nociceptive, inflammatory, and neuropathic pain, either alone or in combination, and that different pain pathways may be responsible for the different types of pain experienced by women because of endometriosis. $^{16}$

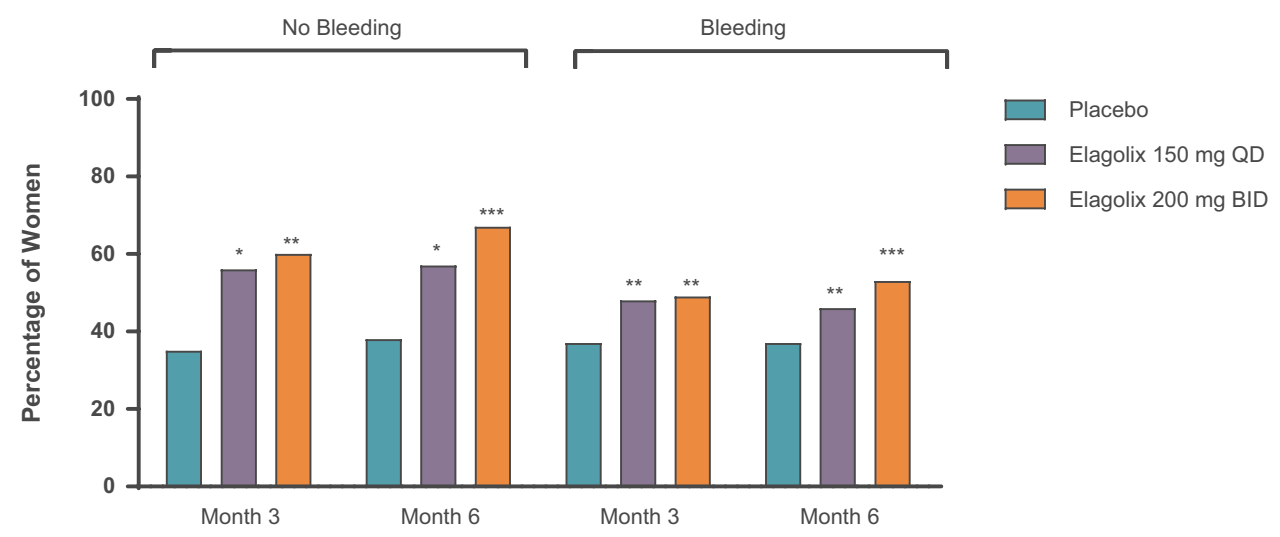

Figure 3 Percent of nonmenstrual pelvic pain responders by bleeding status. Responder equals reduced nonmenstrual pelvic pain and reduced or stable rescue analgesic use. $\mathrm{p}<0.001(* * *), \mathrm{p}<0.01(* *)$, and $\mathrm{p}<0.05(*)$ vs placebo using a contrast within the subgroup in the logistic regression model.

Abbreviations: BID, twice daily; $\mathrm{QD}$, once daily. 
Table 2 Proportion of Simultaneous Nonmenstrual Pelvic Pain Nonresponder and Dysmenorrhea Responder Change from Baseline by Month

\begin{tabular}{|l|l|l|l|}
\hline Month & Placebo & Elagolix I50 mg QD & Elagolix 200 mg BID \\
\hline Elaris EM-I & & & \\
Month 3 & $18(4.8)$ & $33(13.3)$ & $60(24.6)$ \\
Month 6 & $30(8.1)$ & $30(12.1)$ & $44(18.1)$ \\
\hline Elaris EM-II & & & \\
Month 3 & $24(6.8)$ & $23(10.4)$ & $51(22.7)$ \\
Month 6 & $21(5.9)$ & $21(9.5)$ & $48(21.3)$ \\
\hline
\end{tabular}

Notes: Last observation carried forward; intent-to-treat population. Data are presented as $\mathrm{n}(\%)$.

Abbreviations: BID, twice daily; QD, once daily.

Results from this current study have shown that elagolix reduces endometriosis-associated pain in women, both during bleeding and nonbleeding days and despite the presence of bleeding episodes. Although the mechanism of how elagolix interferes with endometriosis-associated pain has not been elucidated to date, it is possible that it exerts its effect through various pain pathways. Findings from a post hoc analysis of the Elaris EM-I and EM-II studies support this hypothesis. In the study by Taylor et al, ${ }^{11}$ improvements observed in women with endometriosis treated with elagolix varied according to different types of pain. The greatest improvements in dysmenorrhea scores were seen between treatment months 0 and 2, after which scores plateaued. ${ }^{11}$ It is possible that the effect of elagolix on dysmenorrhea may be due to its role in reducing estrogen levels, thereby affecting the regulation of peripheral nerve fibers responsible for eliciting pain. Additionally, the effect of elagolix on dysmenorrhea may be due to its impact on prostaglandin secretion, which has been demonstrated to be excessive in the endometrium and in endometriotic lesions during menstruation, leading to abnormal uterine contraction. ${ }^{17}$

The plateau for nonmenstrual pelvic pain was achieved later, with the greatest improvements in pain between months 0 and 4 , and then plateauing after month 4 . Dyspareunia pain scores plateaued after month 4 similarly to nonmenstrual pelvic pain, which suggests disease modification effects by elagolix, for example, a reduction in chronic inflammation and/or central sensitization, or other mechanisms of pain. ${ }^{11}$

As women experience endometriosis-associated pain uniquely, future studies elucidating the mechanism of action of elagolix in women with endometriosis would better our understanding of endometriosis-associated pain and provide insight into the individualization of treatment.
Limitations of this study pertain to the measurement of bleeding days and a placebo effect. Although bleeding days were not objectively measured, they were measured more extensively than in previous studies of endometriosis-related pain. Another limitation is that we were unable to assess whether there were differences in pelvic pain scores between menstruating and nonmenstruating women, given the difficulty in following menstrual patterns at the patient level. Additionally, women in the placebo-treated group also reported a decrease in pain during both bleeding and nonbleeding days. It can be suggested that women would be more likely to report pain if bleeding occurs; therefore, given that women in the placebo group are reporting less pain during bleeding, we can infer that the reduction of pain seen in the elagolixtreatment groups compared with the placebo group during bleeding is more remarkable.

\section{Conclusions}

To our knowledge, this is the first time that reduction in pain associated with endometriosis has been achieved, despite the presence of continued bleeding episodes. This, along with the identification of the mechanisms of action of elagolix through various pain pathways yet to be elucidated, will provide insights into additional treatment options for women with endometriosis-associated pain.

\section{Data Sharing Statement}

AbbVie is committed to responsible data sharing regarding the clinical trials we sponsor. This includes access to anonymized, individual, and trial-level data (analysis data sets), as well as other information (eg, protocols and Clinical Study Reports), as long as the trials are not part of an ongoing or planned regulatory submission. This includes requests for clinical trial data for unlicensed 
products and indications. This clinical trial data can be requested by any qualified researchers who engage in rigorous, independent scientific research, and will be provided following review and approval of a research proposal and Statistical Analysis Plan (SAP) and execution of a Data Sharing Agreement (DSA). Data requests can be submitted at any time and the data will be accessible for 12 months, with possible extensions considered. For more information on the process, or to submit a request, visit the following link: https://www.abbvie.com/our-science/clini cal-trials/clinical-trials-data-and-information-sharing/dataand-information-sharing-with-qualified-researchers.html.

\section{Ethical Approval}

Elaris EM-I and Elaris EM-II studies were conducted in accord with International Conference on Harmonization guidelines and applicable regulations and ethical principles of the Declaration of Helsinki. Shulman Associates IRB (Cincinnati, OH) conducted the majority of the IRB approvals (EM-I/M12-665 IRB approval number 201202559; approval date, April 11, 2012; EM-II/M12671 IRB approval number 201208471; approval date, November 16, 2012).

\section{Informed Consent to Participate}

Informed consent was obtained from all women included in the study.

\section{Informed Consent to Publish}

Written informed consent was obtained from the patient(s) for their anonymized information to be published in this article.

\section{Acknowledgments}

AbbVie and the authors thank the women who participated in this clinical trial and all study investigators for their contributions. Erin Franceschini, MS, and Amy M Spiegel, $\mathrm{PhD}$, both employees of AbbVie Inc., and Michelle Roberts, PhD, and Kersten Reich, MPH, CMPP, of JB Ashtin, provided medical writing support for this manuscript. These data were presented at the 2018 Society of Endometriosis and Uterine Disorders Congress in Florence, Italy, from April 25-28, 2018, and the 2018 Canadian Society for the Advancement of Gynecologic Excellence, 3rd Annual Conference in Banff, Alberta, Canada, from September 27-29, 2018.

\section{Author Contributions}

All authors were involved in data interpretation and critical review of the manuscript content. K.C. and K.G. were involved in the concept/design of the study, and Y. M. performed statistical analyses. The authors and AbbVie reviewed and approved the manuscript; however, the authors maintained control over the final content. The authors had access to relevant aggregated study data and other information (such as study protocol and analytic plan validated data tables; and the clinical study report) required to understand and report research findings. The authors take responsibility for the presentation and publication of the research findings, have been fully involved at all stages of publication and presentation development, and are willing to take public responsibility for all aspects of the work. All individuals included as authors and contributors who made substantial intellectual contributions to the research, data analysis, and publication or presentation development are listed appropriately. The role of the sponsor in the design, execution, analysis, reporting, and funding is fully disclosed. The authors' personal interests, financial or nonfinancial, relating to this research and its publication have been disclosed. All authors meet the International Committee of Medical Journal Editors (ICMJE) criteria for authorship for this article, take responsibility for the integrity of the work as a whole, and have given their approval for this version to be published.

\section{Funding}

AbbVie Inc. funded this study and participated in the study design, research, analysis, data collection, interpretation of data, reviewing, and approval of the manuscript for publication.

\section{Disclosure}

S.K.A. was a study investigator, has received research support from AbbVie and Sobi, and has served on the speakers' bureau for AbbVie. S.K.A. reports personal fees from AbbVie and Myovant. S.S.S. was a study investigator in therapeutic trials for endometriosis and fibroids sponsored by Allergan, AbbVie, and Bayer, and served as a speaker and advisor for Allergan, AbbVie, Bayer, Hologic, Myovant, and Cooper Surgical. D.F. A. was a study investigator and has received grant support from Actavis and Glenmark; research support from Myovant, Mithra, and ObsEva; grant support, 
honoraria, and travel support from Bayer Healthcare, Endoceutics, Merck, Radius Health, Shionogi, and TherapeuticsMD; honoraria and travel support from Exeltis/CHEMO France, Pfizer, Sermonix Pharmaceuticals, and TEVA/HR Pharma; research support and consulting fees from AbbVie; and honoraria and travel support from Agile Therapeutics and Innovagyn. He also has an equity interest in Agile Therapeutics and an equity interest in Innovagyn. Y. M. and K.G. are AbbVie employees and have stock or stock/options. K.C. was an employee of AbbVie Inc. at the time the study was conducted and owns stock. K.C. also reports patent for "Methods of treating heavy menstrual bleeding" (PCT/US2018/043321). E.S. was a study investigator, has received research support from AbbVie, and has served on medical advisory boards and speakers' bureaus for AbbVie, Ferring, and DOT Labs. The authors report no other conflicts of interest in this work.

\section{References}

1. Fuldeore MJ, Soliman AM. Prevalence and symptomatic burden of diagnosed endometriosis in the United States: national estimates from a cross-sectional survey of 59,411 women. Gynecol Obstet Invest. 2017;82(5):453-461. doi:10.1159/000452660

2. Burney RO, Giudice LC. Pathogenesis and pathophysiology of endometriosis. Fertil Steril. 2012;98(3):511-519. doi:10.1016/j. fertnstert.2012.06.029

3. Giudice LC. Clinical practice endometriosis. N Engl J Med. 2010;362 (25):2389-2398. doi:10.1056/NEJMcp1000274

4. Johnson NP, Hummelshoj L, Adamson GD, et al. World Endometriosis Society consensus on the classification of endometriosis. Hum Reprod. 2017;32(2):315-324. doi:10.1093/humrep/dew293

5. Bloski T, Pierson R. Endometriosis and chronic pelvic pain: unraveling the mystery behind this complex condition. Nurs Womens Health 2008;12(5):382-395. doi:10.1111/j.1751-486X.2008.00362.x
6. Greene R, Stratton P, Cleary SD, Ballweg ML, Sinaii N. Diagnostic experience among 4334 women reporting surgically diagnosed endometriosis. Fertil Steril. 2009;91(1):32-39. doi:10.1016/j. fertnstert.2007.11.020

7. Soliman AM, Coyne KS, Zaiser E, Castelli-Haley J, Fuldeore MJ. The burden of endometriosis symptoms on health-related quality of life in women in the United States: a cross-sectional study. J Psychosom Obstet Gynaecol. 2017;38(4):238-248. doi:10.1080/ 0167482X.2017.1289512

8. Soliman AM, Yang H, Du EX, Kelley C, Winkel C. The direct and indirect costs associated with endometriosis: a systematic literature review. Hum Reprod. 2016;31(4):712-722. doi:10.1093/humrep/dev335

9. Zito G, Luppi S, Giolo E, et al. Medical treatments for endometriosis-associated pelvic pain. Biomed Res Int. 2014;2014:191967. doi:10.1155/2014/191967

10. Simpson PD, McLaren JS, Rymer J, Morris EP. Minimising menopausal side effects whilst treating endometriosis and fibroids. Post Reprod Health. 2015;21(1):16-23. doi:10.1177/2053369114568440

11. Taylor HS, Giudice LC, Lessey BA, et al. Treatment of endometriosis-associated pain with elagolix, an oral GnRH antagonist. $N$ Engl $J$ Med. 2017;377(1):28-40. doi:10.1056/ NEJMoa1700089

12. Klein CE, Carter DC, Ng J, Chwalisz K. Dose-dependent suppression of gonadotropins and ovarian hormones by elagolix in healthy premenopausal women. J Clin Endocrinol Metab. 2017;102(5):1683-1691

13. Donnez J, Taylor HS, Taylor RN, et al. Treatment of endometriosis-associated pain with linzagolix, an oral gonadotropin-releasing hormone-antagonist: a randomized clinical trial. Fertil Steril. 2020;114(1):44-55. doi:10.1016/j. fertnstert.2020.02.114

14. Ng J, Klein CE, Carter DC, Chwalisz K. Dose-dependent suppression of gonadotropins and ovarian hormones by elagolix in healthy premenopausal women. J Clin Endocrinol Metab. 2017;102(5):16831691. doi:10.1210/jc.2016-3845

15. Morotti M, Vincent K, Becker CM. Mechanisms of pain in endometriosis. Eur J Obstet Gynecol Reprod Biol. 2017;209:8-13. doi:10.1016/j.ejogrb.2016.07.497

16. Vercellini P, Viganò P, Somigliana E, Fedele L. Endometriosis: pathogenesis and treatment. Nat Rev Endocrinol. 2013;10(5):261. doi:10.1038/nrendo.2013.255

17. Sacco K, Portelli M, Pollacco J, Schembri-Wismayer P, Calleja-Agius J. The role of prostaglandin E2 in endometriosis. Gynecol Endocrinol. 2012;28(2):134-138. doi:10.3109/09513590.2011.588753
Journal of Pain Research

\section{Publish your work in this journal}

The Journal of Pain Research is an international, peer reviewed, open access, online journal that welcomes laboratory and clinical findings in the fields of pain research and the prevention and management of pain. Original research, reviews, symposium reports, hypothesis formation and commentaries are all considered for publication. The manuscript management system is completely online and includes a very quick and fair peer-review system, which is all easy to use. Visit http:// www.dovepress.com/testimonials.php to read real quotes from published authors. 